\title{
ETNOGRAFÍA URBANA EN SALAMANCA: USO, ORIGEN Y AUTENTICIDAD DE LA BALDOSA HEXAGONAL DE GAUDÍ EN LA PROVINCIA DE SALAMANCA
}

\author{
Juan Ignacio García Hernández ${ }^{1}$ y Rosalía Hdez. Pérez de Burgo ${ }^{2}$ \\ "!Interesante! Joven distinguido, de extensa cultura y \\ conocimientos de arqueología e historia, desde el siglo \\ XI a la edad contemporánea, de fácil y brillante estilo \\ literario, se ofrece para redactar la historia de los estudios \\ generales del orbe, París, Oxford, Bolonia, etcétera, \\ desde su fundación hasta la dominación francesa. Darán \\ razón en la venta del desahogo, camino del egoísmo, a \\ mano derecha según se va hacia el presupuesto."
}

Libertad. Periódico semanal. Año I nº 19. 26 de Junio de 1913

\section{Resumen:}

Ofrecemos en este documento un breve recorrido por la técnica y la historia de la baldosa hidráulica en su versión más decorativa: el mosaico hidráulico y el panot hexagonal de Gaudí. El objetivo es, por un lado, hacer hincapié en la necesidad e importancia de estos estudios dedicados a la arqueología industrial y etnografía urbana de los últimos dos siglos. Por otro, y, en consecuencía de lo enunciado, hablaremos aquí de la imitación del panot hexagonal diseñado originalmente por Gaudí utilizado en un conocido hotel de la provicia de Salamanca.

Palabras claves:

Arqueología industrial. Antoni Gaudí. Baldosa hidráulica. Etnografía urbana. Salamanca.

\section{Abstract:}

We offer in this paper a brief tour to the art and technique of the hydraulic tile in its most decorative version: hidraulic mosaics and the hexagonal tile of Gaudí. The aim is -in one hand- to emphasize the concept of industrial archeology to promote all this products wich have being manufacturated in the last two centuries. In other hand, we will identificate here as an imitation of that hexagonal Gaudi's tiles used for outdoor decoration in a popular hotel in the province of Salamanca.

\section{Key words:}

Industrial archeology. Antoni Gaudí. Hydraulic tile. Urban etnography. Modernism. Salamanca.

1 Arqueólogo/Gestor de Patrimonio Cultural - arqueoart@hotmail.com

2 Restauradora y conservadora de Bienes Culturales Artista multidisciplinar geometricadomestica@gmail.com

Revista Otarq - ISSN 2530-4933

Vol. 2 2017, pp. 53-74 


\section{Introducción ${ }^{3}$.}

Por lo general, el estudio en profundidad de los pavimentos por parte de disciplinas como la Arqueología y la Historia se centra en el mosaico romano en cuanto a solados domésticos y a los empedrados medievales en lo que a suelo público se refiere. Sus diseños, su fabricación, sus métodos de colocación.

Reclamamos el mismo ahínco investigador por técnicas modernas que forman ya parte de nuestro patrimonio industrial más reciente: las baldosas hidráulicas en sus variantes de mosaico hidráulico (interior) y la baldosa hexagonal en relieve de Gaudí. En apariencia diferentes, tienen en común su marcada intención decorativa utilizando un material en principio basto y con un valor muy inferior al de la cerámica, la madera o la piedra: el cemento Portland prensado con máquina hidráulica.

En el presente documento trataremos estos asuntos además de identificar como una imitación el enlosado con baldosa o panot hexagonal de Gaudí empleado en el Complejo Hotel Regio S.L.U. sito en la Carretera Salamanca-Ávila-Madrid, km 4. 37900 Santa Marta de Tormes. Salamanca.

\section{Cuestiones preliminares}

\section{La baldosa hidráulica}

Tanto la baldosa o mosaico hidráulico como la baldosa hexagonal de Gaudí son posibles gracias a la invención y desarrollo de su material de base: El cemento Portland.

El cemento Portland debe su nombre a su parecido con la piedra Portland, típica de la Isla de Portland, en Dorsey, Inglaterra. Fue patentado en 1824 en Inglaterra por Joseph Aspdin ${ }^{4}$, aunque el mérito no es sólo suyo ya que otros fabricantes e investigadores ya estaban trabajando para mejorar la composición que haría del cemento romano un material nuevo, más resistente a la humedad y más rentable. Su éxito se debe a varios descubrimientos muy seguidos en el tiempo:

3 No podemos dejar de expresar en estas líneas el agradecimiento y gratitud a ciertas personas y organismos, los cuales nos brindaron la posibilidad de consultar la información requerida, suministrarnos información relacionada, o encauzarnos en diferentes propósitos. Por tanto, agradecemos su ayuda a, D. Miguel García Figuerola, Director del Museo del Comercio de Salamanca; a Enric Pericas Bosch, Casa Escofet; a D. Marcelino Martín Moreno, Director del Hotel Regio; a Da. Lourdes Raymundo Prevost, Arquitecto, secretario técnico del COAL Delegación de Salamanca; al COAL -Colegio Oficial de Arquitectos de León- Delegación de Salamanca; a D. David Senabre López, profesor de la cátedra de Geografía de la Universidad Pontificia de Salamanca UPSA; a Montserrat Morenilla, de la Fundación DOCOMOMO Ibérico.

4 J. Aspdin. 1788-1855. Propietario de la primera patente de cemento Portland. 
- La invención de altos hornos industriales ${ }^{5}$ que permitían calcinar el material base a mucha más temperatura y de manera constante.

- La correcta mezcla de los áridos obtenidos con cal hidráulica ${ }^{6}$.

- En lo que a baldosa hidráulica se refiere, es fundamental añadir:

- La prensa hidráulica

- El abaratamiento de coste que suponía fabricar baldosas sin necesidad de una última cocción como ocurría con los materiales cerámicos.

Los pavimentos domésticos de estilo medieval, realizados entonces con cerámica-gres, están de moda en esta época. Realizarlos con mosaico hidráulico supone un coste menor, por lo tanto vamos a encontrar ejemplos cada vez más ricos y complejos en cuanto a diseño y colocación. Desde la baldosa cuadrada de $20 \times 20 \mathrm{~cm}$ hasta los mosaicos Nolla, llenos de detalles.

Gracias a su fabricación semi manual cada baldosa es única, igual y diferente del resto que componen el pavimento.

La baldosa hidráulica está compuesta de tres capas diferenciadas:

- Capa 1: Es la capa visible de la baldosa. Está formada por una pasta fluida de cemento Portland blanco ( a veces gris), una pequeña cantidad de polvo de mármol y colorante o pigmento. Esta pasta se distribuye a mano por las zonas de color delimitadas en el molde de acero, también Ilamado trepa o plantilla de metal. Tiene un grosor de $4 \mathrm{~mm}$ aprox.

- Capa 2: Esta capa intermedia se denomina brasage. Sobre la capa anterior, previa retirada de la trepa, se aplica una mezcla de arena y cemento Portland en seco. Su función es absorver el agua de la capa anterior. Puede llegar al centímetro de grosor.

- Capa 3: El gros. Compuesta por arena y cemento Portland, similar a la capa anterior, pero de una calidad inferior que abarata el coste de producción y al mismo tiempo proporciona una mejor adherencia de la baldosa al mortero al conformar una superficie mucho más rugosa.

\footnotetext{
5 Derivados del modelo de F.G.Hoffman patentado en 1858.

6 J.L. Vicat como investigador de la cal hidráulica. W. Michaelis y Le Chatelier como desarrolladores de la fórmula, entre 1870 y 1880, fueron fundamentales para el desarrollo de este material hasta que en 1878 la Asociación de Fabricantes de Cemento estableció un estándar para el cemento Portland basado en sus estudios.

7 William Boulton patenta la primera prensa hidráulica para fabricar baldosas en 1868 .
} 


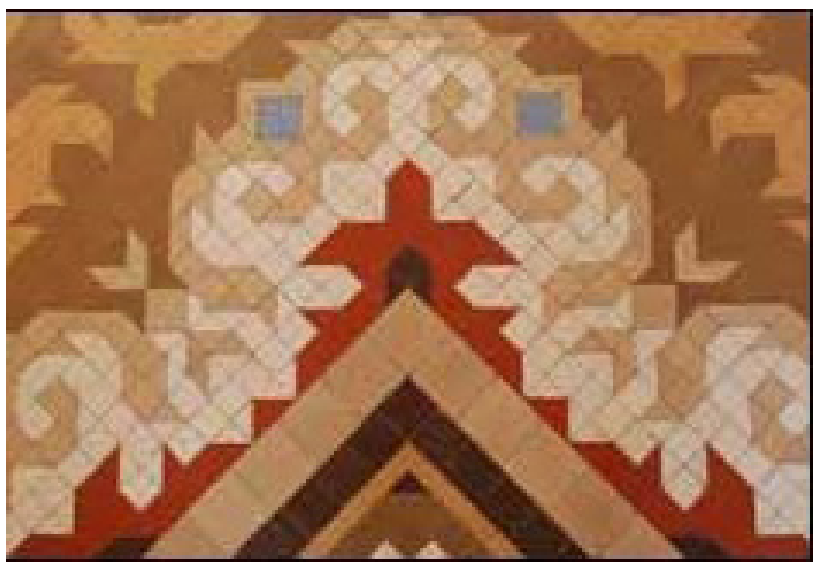

Fig. 1: Mosaico Nolla.

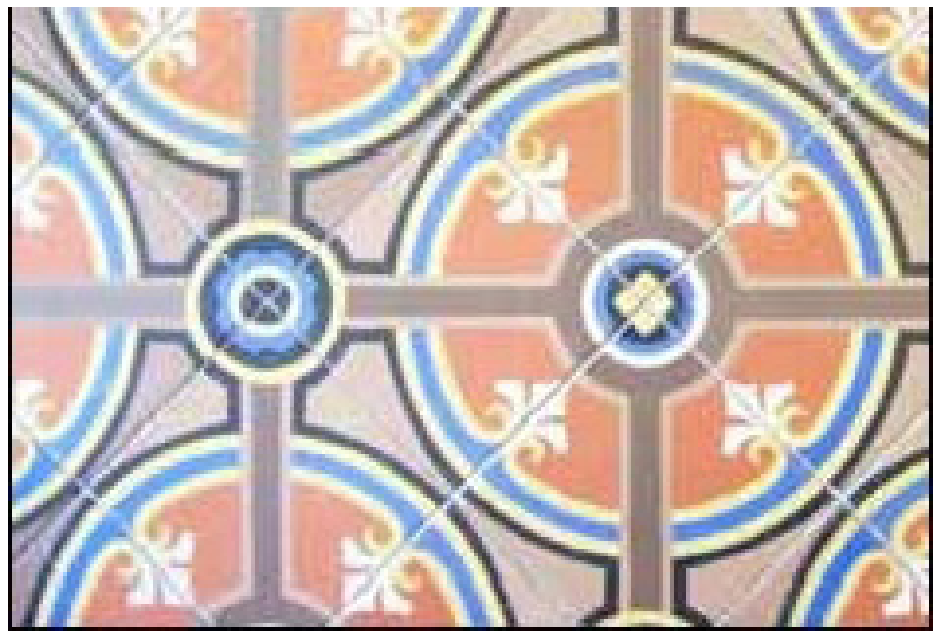

Fig. 2: Mosaico en baldosa.

Así, después de un conveniente alisado, se aplica el golpe de presión necesario para que se unan todos los estratos con una prensa hidráulica que trabaje como mínimo a $120 \mathrm{~kg}$ por $\mathrm{cm}^{2}$ aunque puede variar, según la calidad y el fabricante, hasta los $90 \mathrm{~kg}$ por $\mathrm{cm}^{2}$.

Finalmente se colocan las baldosas en parrillas fuera de su molde y se dejan secar durante semanas. En España, las fábricas más importantes dedicadas a este producto se encontraban en Cataluña. En la Exposición Universal de París de 1867 aparecen varias compañías de Barcelona: Rivet Compañía, Osola Company, Sola y Cía., Fortuny Cía., que desarrollaban en aquel momento diseños de estilo Art Nouveau o Modernista ${ }^{8}$.

8 Dentro de nuestra provincia, uno de los grandes arquitectos de la ciudad, Joaquín de Vargas, 
Hasta 1920 este tipo de pavimento se considera la última moda. Después, va perdiendo importancia hasta que en 1950 decae por completo para ser sustituido por materiales más ligeros y/o más económicos.

En la actualidad, aún existen fábricas en España, Francia y Marruecos que mantienen viva esta moderna tradición con bellos patrones tanto antiguos como nuevos y con una gran calidad derivada de la evolución natural de la industria y la tecnología.

\section{La baldosa hidráulica de Gaudí}

Este tipo de baldosa hidráulica se conoce en construcción con el nombre más concreto de panot $^{9}$, generalmente son cuadradas, en relieve para evitar deslizamientos, pues se sitúan preferentemente al exterior o en la vía pública. La particularidad de la que nos ocupa es, entre otras cosas a desarrollar en este capítulo, su forma hexagonal y su bella decoración con motivos del fondo marino. Las formas poligonales están presentes en toda la obra de Gaudí, ya fuera como parte determinante de los espacios o elementos constructivos, o como generadores - o generando decoración. El polígono plano regular más usual en las obras de Gaudí es el hexágono, descubriendo la posibilidad dentro de ellos de diferentes divisiones, ya sea la unión de 3 rombos o la confluencia de 12 triángulos rectángulos. Incluso, utilizando la forma en sí enmarcando los motivos dentro de una perspectiva caballera. Cualquiera de estas posibilidades son recurrentes en toda la obra de Gaudí. La baldosa hexagonal de Gaudí, inicialmente pensada para pavimentar el suelo de la Casa Batlló, y que más tarde se utilizó en las habitaciones de la Casa Milá, es una baldosa inscrita en un hexágono regular, con unas medidas de $144 \mathrm{~mm}$ de lado, $250 \mathrm{~mm}$ de diámetro, y $45 \mathrm{~mm}$ de grosor, de textura en bajorrelieve.

Las características técnicas de este panot son las mismas con las que fue diseñado, es decir:

realiza un artículo periodístico (Adelantos Industriales. Construcción. Noticiero Salmantino: diario imparcial de la tarde. Año V número 1563. 3 de julio de 1902) donde habla de los adelantos industriales dentro del mundo de la construcción. Uno de esos adelantos, son las baldosas de hormigón comprimido "De poco tiempo a esta parte se han empezado a utilizar en la pavimentación de las calles de Londres baldosas de hormigón que se consiguen por medio de un ingenioso aparato,.." <http://prensahistorica.mcu.es> .

9 [del fr. panneau, íd.] m CONSTR/OBR PÚBL Lloseta feta amb morter de ciment i sorra destinada a fer paviments resistents, especialment els situats a la intempèrie. <http://www.enciclopedia.cat/>; En castellano se traduciría como loseta, baldosa, losa [del celtolat. lausia, losa, voz de or. Hisp] f. Piedra llana y de poco grueso, casi siempre labrada, que sirve para solar y otros usos. < http:// buscon.rae.es/ drael/>. Aprovechamos estas líneas para romper una lanza a favor del termino panot para referirnos a este tipo de pavimento. 

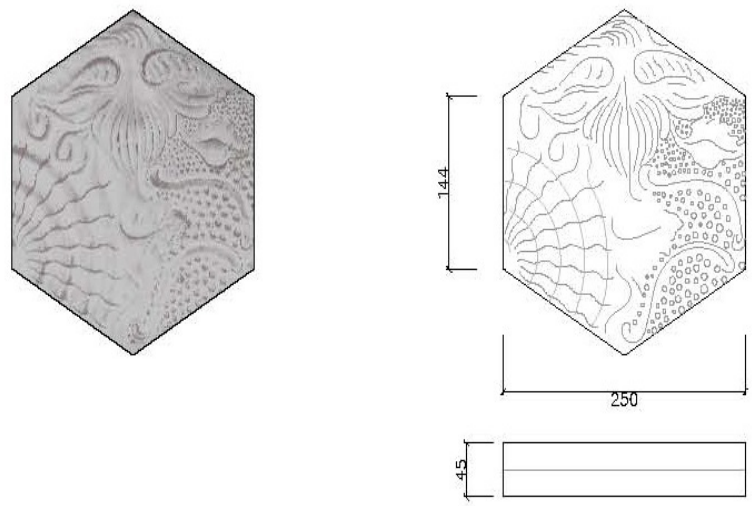

Fig. 3: Características técnicas de la baldosa ${ }^{10}$.

Según palabras de Daniel Giralt-Miracle, comisario general del Año Internacional Gaudí 2002 en Barcelona¹1:

"De todas las obras no arquitectónicas realizadas por Gaudí, la de mayor fortuna, la más editada y probablemente la más popular sea la baldosa hexagonal que el arquitecto proyectó en 1904 para pavimentar el suelo de la Casa Batlló, un edificio de viviendas promovido por el industrial textil Josep Batló en el Paseo de Gracia barcelonés en el que el concepto del mar y del agua es omnipresente. En este edificio Gaudí llevó a cabo una intervención integral, ya que diseñó todos los elementos arquitectónicos, de mobiliario y ornamentales, incluido el pavimento, que finalmente por un retraso en la producción no se pudo aplicar. Por este motivo Gaudí, decidió usar estas baldosas en las habitaciones de servicio de la Casa Milá (La Pedrera), en inmueble que proyectó inmediatemente después, también en el Paseo de Gràcia. La denominada "baldosa Gaudi" es un buen ejemplo de la manera de proceder del arquitecto, ya que en ella confluyen dos de las constantes de su obra: la vertiente geométrica y la simbólica. Tanto la forma de la baldosa como las imágenes que representa nos remiten a la filosofía gaudiniana, que se basa en la observación meticulosa de las morfologías y las estructuras de la naturaleza, "esta naturaleza que siempre ha sido mi maestra". Si los perfiles de seis lados nos recuerdan las celdas de los ojos de ciertos insectos o las de un panal de abejas, los caparazones de las tortugas o la piel de cocodrilo, las figuras que aparecen en las baldosas,

$10<\mathrm{http} / / / w w w . e s c o f e t . c o m>$

$11<$ http://www.panotgaudi.com/la-baldosa> 
una estrella de mar, una caracola y una medusa, evocan animales sinuosos y en movimiento procedentes del mundo marino.

La prodigiosa capacidad en Gaudí de ver las formas y combinarlas se pone de manifiesto una vez más en esta propuesta, que solamente es comprensible cuando se juntan siete piezas hexagonales, ya que cada una sólo muestra un tercio de los símbolos dibujados en un suave bajo relieve, un planteamiento que rompe con la tradición de la baldosa cuadrada que tiene un único motivo ornamental".

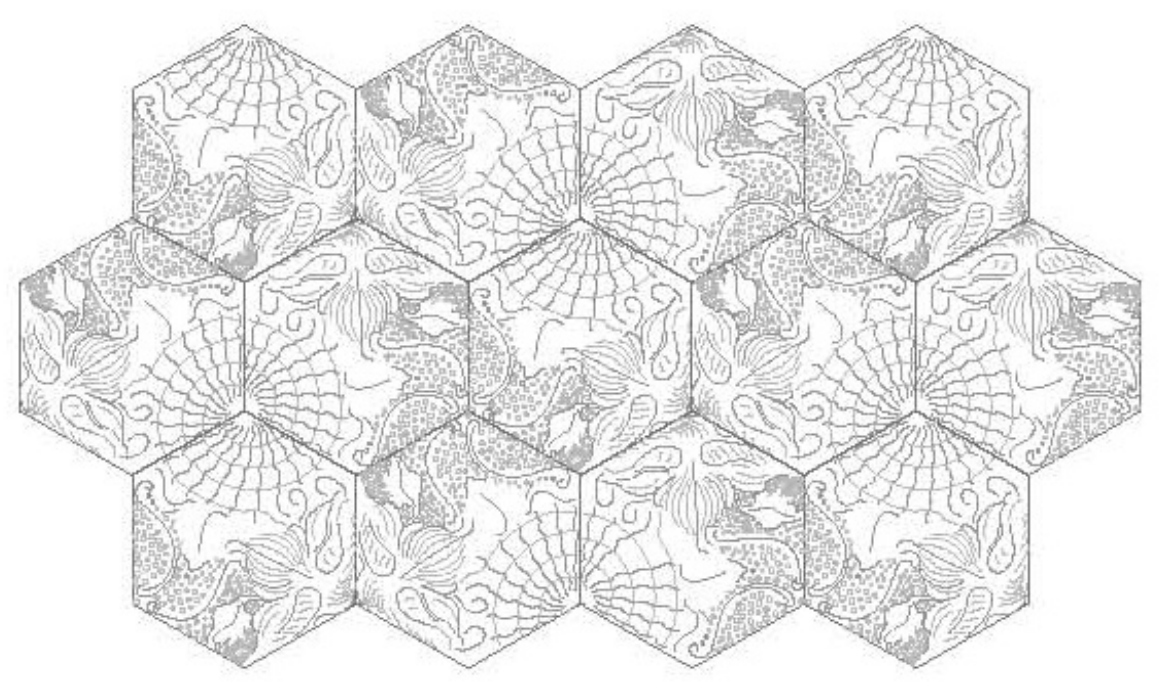

Fig. 4: Estructura combinada de baldosas.

Como bien comentan los diferentes autores, la visión de los motivos decorativos de estas baldosas, solamente son apreciables cuando se combinan -a partir de 7 piezas- donde preciamos los tres motivos diferentes que aparecen en cada tercio de la baldosa.

Los motivos que aparecen con la unión de las baldosas son tres formas diferenciadas perfectamente unidas, de corte marino, observándose: una forma esférica, casi espiral -cuadrado rojo- a modo de una caracola, incluso podríamos decir que más tirando a un ammonite, de simbología marina; una forma triangular o hexagonal - cuadrado verde- a modo de medusa, o motivo vegetal, de igual simbología; y otra forma circular o de tipo espiral-cuadrado azul-asemejándose a una estrella de mar -simbología marina de nuevo ${ }^{12}$ - o a algún motivo vegetal de tipo floral.

12 Aunque sabemos perfectamente que la intención del autor de utilizar una simbología marina para los motivos de las baldosas, nos es razonable comentar como esta forma se asemeja a los motivos circulares de las estelas prerromanas de 6 gamas con giro a la izquierda, vinculados a posibles cultos solares $y / o$ astrales. 


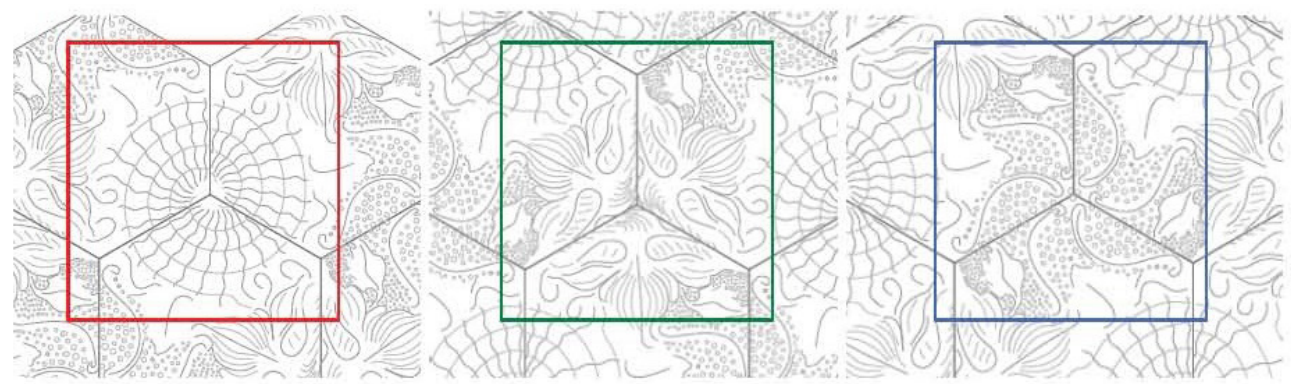

Fig. 5: Detalle de los motivos decorativos.

Todos los motivos están realizados con una proyección de plano cenital, pero la forma de moldear la baldosa cercana al bajorrelieve, le da ese carácter sinuoso y de movimiento pendulante, como si anduviéramos sobre las aguas.

Después de su proyección para la Casa Batlló y su posterior uso en la Casa Milá y otros espacios, el molde artesanal con el que trabajó Gaudí se guardó y durante un tiempo no se reparó en él.

En la década de los 70 el Ayuntamiento de Barcelona, aprovechando una profunda remodelación del Paseo de Gràcia con obras de tanta importancia como la construcción de los aparcamientos situados bajo la calzada central, pensó en pavimentar este paseo con los motivos de Gaudí pero, en vez de utilizar el molde original y hacer las baldosas tal y como fueron proyectadas, se realizó una adaptación a serie industrial por parte de Jordi Ros, dibujante/diseñador de la casa Escofet $^{13}$. Para esta adaptación se prefirió como material el vibrazolit ${ }^{14}$, adaptando su relieve y aumentando su tamaño hasta los $43 \mathrm{~cm}$ de los 25 originales del molde de Gaudí.

13 Noticias sobre la casa Escofet en la provincia las encontramos en los años 1897 y 1898 (La opinión. Diario de Salamanca. Época 2a. Año 1. No. 31. 9 Agosto 1897). "Almacén de cerámica, azulejos y pavimentos finos. En este almacén se encontraran todas las clases de productos cerámicos, tales como ladrillos, tejas, impostas, cornisas y aleros,.......... de la gran fabrica la primera de castilla la Vieja, de D. Eloy Silió de Valladolid, pavimentos de todas clases, para salones, salas, portales, etc,..de la de Escofet, Tejera y compañía de Barcelona"; En los periódicos sindicalistas se hacen eco del final de la huelga de los obreros de la casa Escofet en 1908. (El Obrero. Órgano de la Federación Obrera de Salamanca. Año II. № 10. 19 de Septiembre de 1908).

14 En la documentación consultada y prestada por la Casa Escofet para este estudio, se incluyó una nota de envío - fax- donde se podía leer: "Nos complace enviuarles unas idea gráficas de vibrazolit relieve, pavimento para exteriores, que complementa nuestras series pulimentadas de Vibrazo relieve y Vibrazo dibujo. Con el hemos pavimentado ya numerosas calles, aceras, urbanizaciones, andenes, muelles, paseos y estaciones. (una última realización con Vibrazolit, es el paseo de Gracia de Barcelona.)".. 


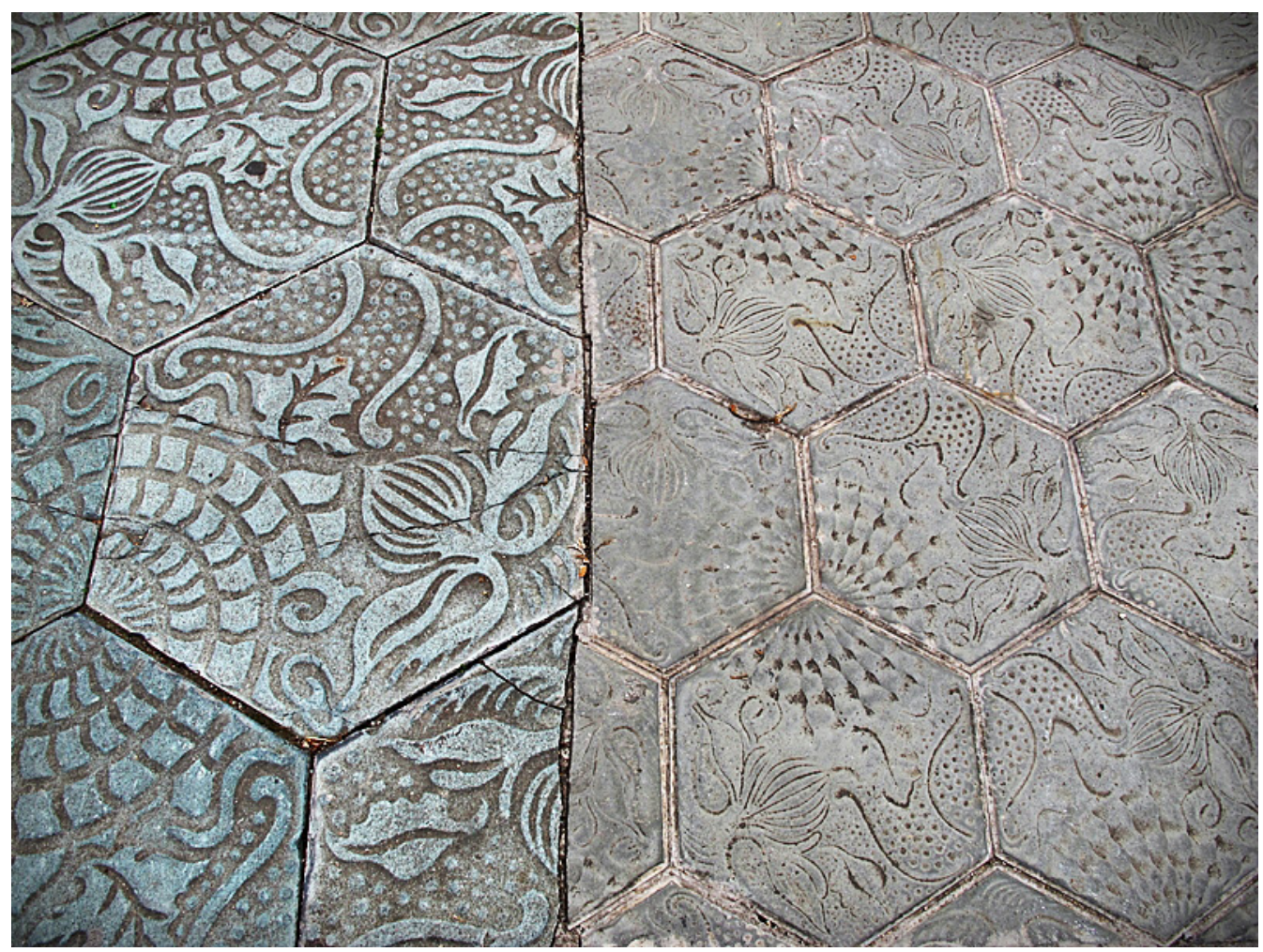

Fig. 6: Visión de los dos pavimentos. Paseo de Gracia, Barcelona. ${ }^{15}$

Este pavimento -de mayor tamaño, mayor relieve- estuvo comercializándose por toda la geografía española desde la época de los 70, realizándose diferentes obras de pavimentación de gran envergadura, como la que se realizó en el Paseo de Grao en Castellón, y en otras zonas, como el caso que nos atañe.

En 1997, el Ayuntamiento de Barcelona se propuso volver a pavimentar el paseo de Gracia, pero esta vez se declinó por el tamaño original del panot y por un tratamiento del relieve en negativo, ya que el tráfico rodado había destruido bastantes de las piezas anteriores.

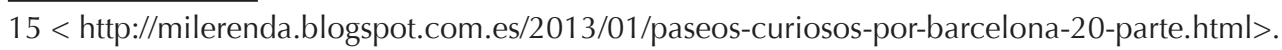




\section{ALGUNOS MODELOS DE VIBRAZO RELIEVE}

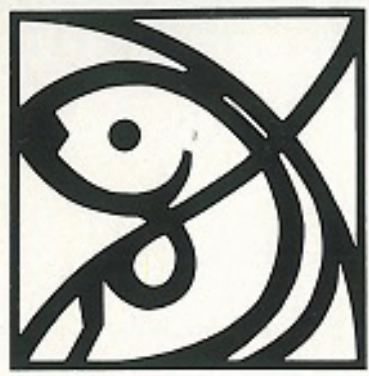

N.' 199

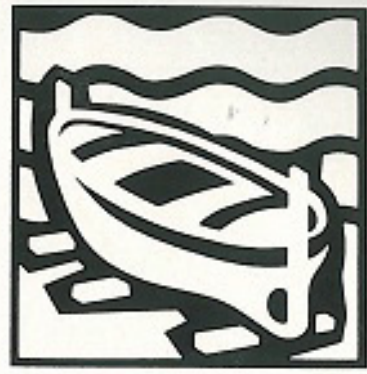

$N \cdot 202$
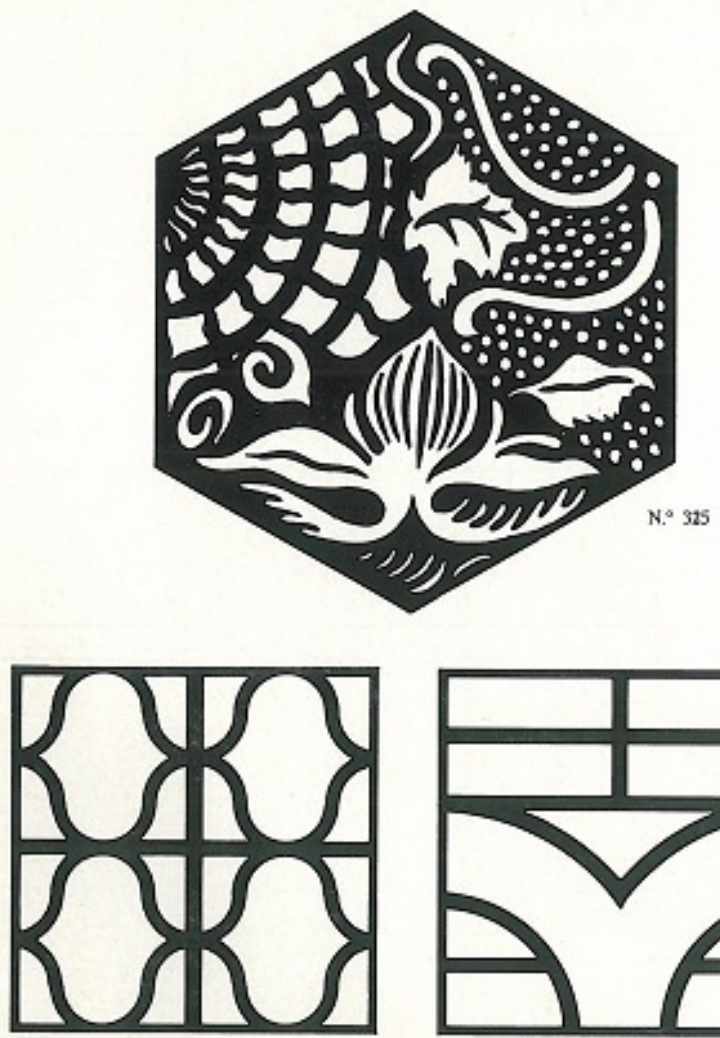

$\mathrm{N}: 550$

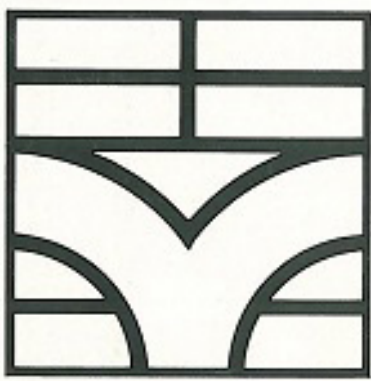

N. 510

Fig. 7: Catalogo Escofet. (1976).

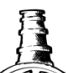




\section{Vibrazolit Relieve para exteriores}

\section{Escotert}

i) Medule $325 / 225$ Exagonal

Disetio origiesl del Arg. Antonio Gaud:

Adopuacion a serie industrial de J. Res.

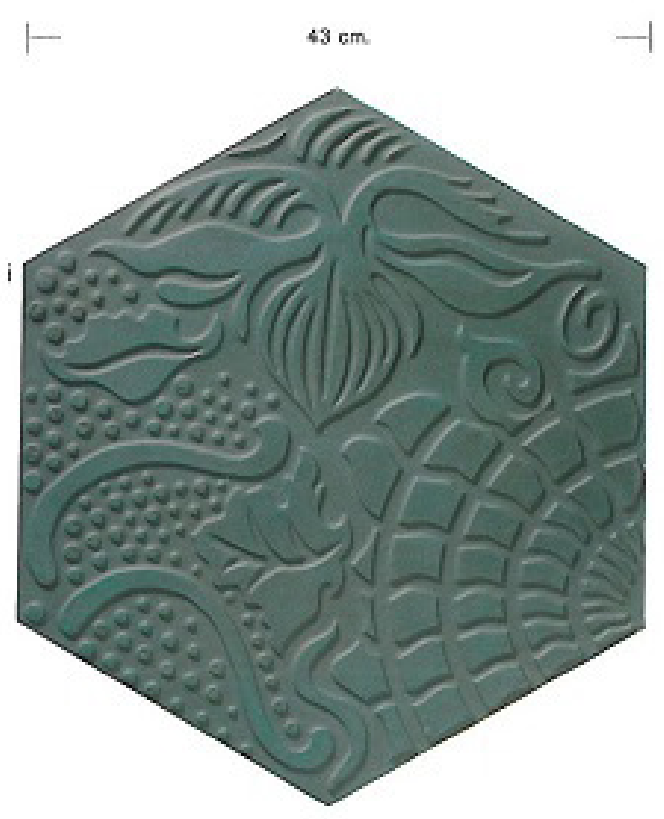

Fig. 8: Catalogo Escofet. (1990).

En las operaciones de sustitución, las losetas grandes eran arrancadas y dejadas en los contenedores de escombros de donde los transeúntes se las Ilevaban como recuerdo, como se podía leer esos días en la prensa ${ }^{16}$. Pero lo que los viandantes no sabían, es que esas losetas no era el panot original moldeado sobre molde de cera a mano por el genial arquitecto, ya que las auténticas son las de menor tamaño. La noticia del cambio de baldosas tuvo su repercusión en la prensa del momento e incluso hubo críticas por parte de la ciudadanía, aludiendo que estaban quitando las originales para poner otro modelo. Como se lee en el catálogo de la casa Escofet, "Al producir el negativo para el espacio público, aumenta sustancialmente la superficie de la cota superior del panot con lo que se asegura la mejor preservación del diseño en en el tiempo y la función antideslizante".

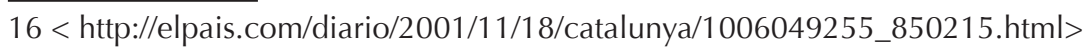



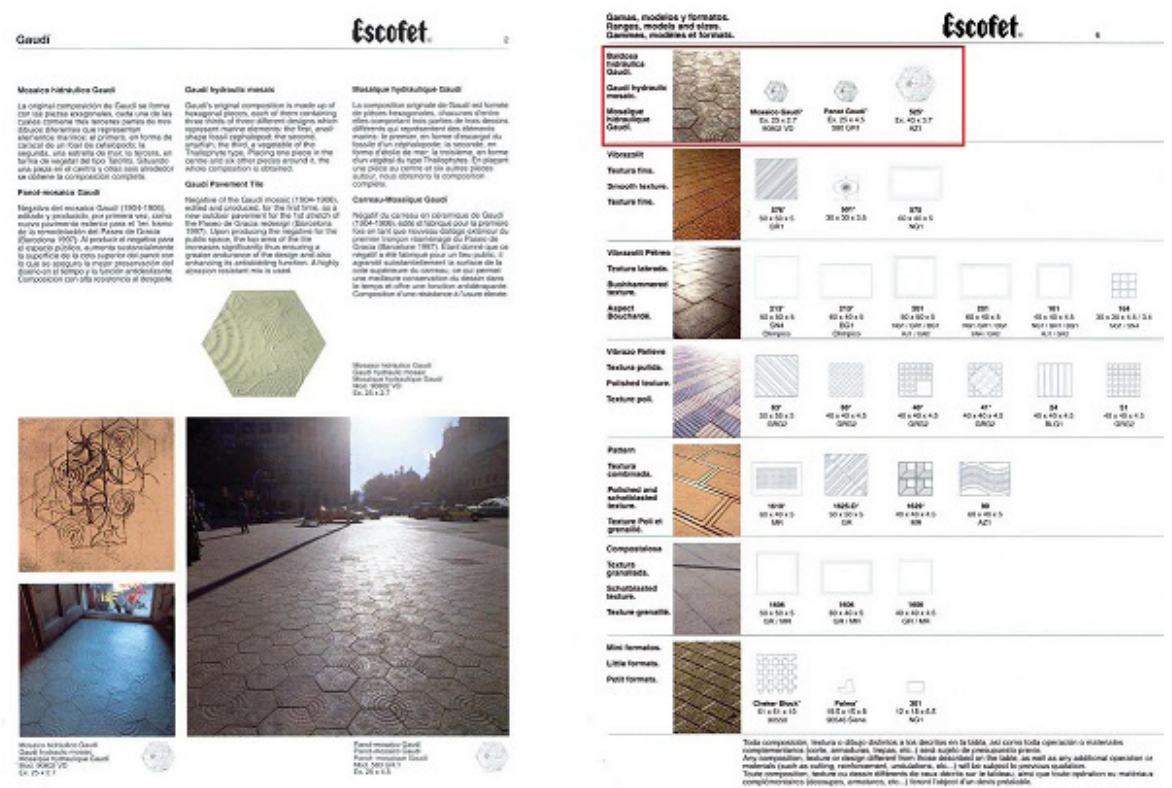

Fig. 9: Catálogo Escofet (2002). Pavimentos urbanos. Gamas, modelos y formatos. Pp.2. en recuadro rojo, gamas, modelo y formatos de la baldosa hidráulica de Gaudí.

Este pavimento -según el modelo de A. Gaudí- estaba pensado tanto para exterior como para interior, y así fue utilizado. Sin embargo, su masiva utilización ha sido al exterior. Destacando lugares donde podemos encontrar este pavimento en sus dos vertientes, recordemos primeramente, la doble pavimentación del paseo de Gracia, en los años 70 y a partir de 1997; el modelo de baldosa de los años 70 lo encontramos en las inmediaciones al museo de Gaudi de la localidad de Reus, en una tonalidad de color rosado; este mismo pavimento está utilizado en el Paseo de Grao de Castellón. Por su parte, en Córdoba, estas baldosas adornan el suelo de la Avenida Conde Vallellano y la Avenida del Corregidor; el pavimento original de Gaudí, lo encontramos -aparte de en La Pedrera- en el Park Güell bordeando una estancia habitacional. En este caso encontramos dos colores diferenciados, tanto gris verdoso como rojizo, observando un refinamiento incluso en los bordes del conjunto; de otra manera lo encontramos en la Alameda de Xátiva, en las escaleras de la Baixada del Carme (Valencia) queda un resto del primer pavimento que se utilizó en época moderna. En este caso son baldosas de cemento de color rojizo, de estructura cuadrangular, en las cuales se puede entrever como motivos centrales las características formas de Gaudí (caracola, medusa y estrella de mar) rodeadas por hexágonos de pequeño tamaño. Cada baldosa, en este caso, es individualizada y no necesita una composición para observar el dibujo. 


\section{Estado de la cuestión}

Después de explicar brevemente la tecnología y la historia de la baldosa hidráulica en su versión más decorativa, nos acercamos al motivo de nuestra disertación: el uso y el origen del panot hexagonal de Gaudí en la pavimentación exterior de un conocido hotel de la provincia helmántica, concretamente en las puertas de acceso a la cafetería y en un patio interior del Hotel Regio, situado en la carretera Ávila - Madrid N-501 en la Localidad de Santa Marta de Tormes, Salamanca.

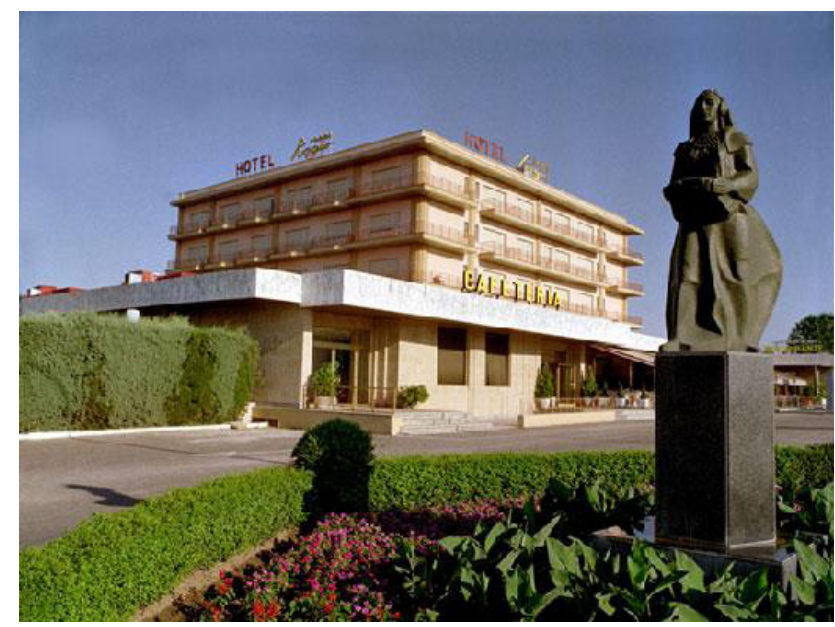

Fig. 10: Vista del complejo.

La notoriedad ${ }^{17}$ o no de estos restos se hace patente al preguntar al personal del hotel sobre las citadas baldosas, las cuales son calificadas como baldosa catalana, sin ninguna otra distinción. Diacrónicamente hablando, por lo que corresponde a la utilización como a la fabricación de la baldosa hidráulica en la provincia, parece ser que hubo diferentes fábricas o artesanos dedicados a este oficio, dividiéndose en ceramistas, alfareros, tejares, etc...Aparte de las diferentes fábricas de áridos, cementos y variados en las cercanías del río y sobre todo en la parte de Tejares $^{18}$, destacando como principales dentro de la especialidad que nos

17 "Nuestras tareas van encaminadas al conocimiento, conservación y difusión del patrimonio, ordenada a la consecución de objetivos sociales que afecten al patrimonio cultural; Gestionarlo, incluye todas las acciones que llevan a ello, por tanto, la gestión del patrimonio (arqueológico) es el conjunto de actuaciones destinadas a hacer efectivo su conocimiento, conservación y difusión, que incluye ordenar y facilitar las intervenciones que en el se realicen". (QUEROL; MARTINEZ DIAZ 1996).

18 Etimológicamente hablando, este barrio de Salamanca -antigua villa- debe su nombre por la cantidad de tejares dedicados a la fabricación de tejas y ladrillos de adobe situados a lo largo 
atañe, la Fábrica de Mosaicos de D. Tomas Martín Bazán ${ }^{19}$, El Taller de Mariño y el Centro Técnico Industrial de Jesús Pérez de la Fuente, representante de la casa Silió de Valladolid, que más tarde cambiaría su nombre por el de "La Juliana", estando activa esta última hasta mediados de los años 80.

Sin embargo, sabemos que existían otra serie de artesanos ceramistas -como podemos apreciar en un escrito literario de primeros de siglo XX, donde aparecen los artesanos Hijos de Prudencio García, Manuel y Jesús Serrano García, y Fernando Romo Barba- sin olvidar otras industrias parejas como La Cerámica Salmantina de Isidoro Sánchez, la Fábrica de Teja y Ladrillo de Salamanca y Tejares, la Cerámica de la Maza de Mariano Muñoz, Manuel y Julián Barba, el Centro Técnico Industrial, Grandes almacenes de materiales para la construcción de edificios de Francisco Pérez Entisne, Fábrica de cerámica de construcción y ornamentación El Carmen la Maza de Alba, entre otros ${ }^{20}$.

Centrándonos en nuestros restos en cuestión se enmarcan dentro de diferentes espacios al exterior del complejo hotelero, llegando a pavimentar una superficie de aproximadamente $270 \mathrm{~m} 2$, situados en la entrada a la cafetería y en una entrada lateral a la misma en la parte Oeste del complejo hotelero -cada porche de unas medidas aproximadas de $20 \mathrm{~m} 2$ - así como en la parte del patio-jardín que se encuentra en la zona Norte del complejo, junto a la linde con la carretera - pavimentando un espacio circundado de aproximadamente $230 \mathrm{~m} 2$. La baldosa que observamos, es una baldosa inscrita en un hexágono regular, con unas medidas de $270 \mathrm{~mm}$ de lado, y $470 \mathrm{~mm}$ de diámetro, realizada en vibrazolit de color grisáceo con capa pictórica de color granate -color vino- con pintas de color negro y blanco. El mortero que constituye su cara vista, de $6 \mathrm{~mm}$ de espesor, está formado por cemento blanco con colorante inorgánico, aditivos y áridos finos especiales, junto con su mortero de base que completa el espesor de la baldosa.

de su perímetro. Destacamos como curiosidad de la importancia del lugar, la existencia de la Colonia Industrial-Agrícola de Vistahermosa, propiedad de D. Saturnino Charro, en el lugar donde actualmente se encuentra el complejo residencial del mismo nombre.

19 La carrera comercial de este industrial terminó del mismo modo que la de gran parte de la sociedad española en la década de los 30. < http://www.salamancamemoriayjusticia.org/vic_ver. asp?id=15111>.

20 Como dato curioso, en un artículo de El Adelanto (año XXVII no 8203 del 18 de Marzo de 1911) dedicado a las industrias salmantinas, realiza un análisis del tejido industrial salmantino, donde resume la industria cerámista: "Existen dos cerámicas, con 25 operarios, nueve horas y media de trabajo, y 1,75, 2,50 y 3 pesetas de jornal; ocho alfarerías, con 24 obreros, nueve horas de trabajo y 2 pesetas de salario, y setenta tejares, con 140 obreros y 2 pesetas de jornal. Tanto por la cantidad como por la calidad de sus productos, los alfareros carecen de importancia. Se limitan a la construcción de vasijas ordinarias de barro cocido. Casi lo mismo sucede con los tejares, en los que se fabrican baldosas, ladrillos y tejas ordinarias, y con arreglo a la venta anual que se hace en los pueblos limítrofes. De esta industria solamente tienen importancia La Cerámica Salmantina, de don Isidoro Sánchez, que fabrica unas 80.000 piezas anuales, y la fábrica de mosaicos de don Tomas Martín Bazan, muy interesante por la perfección de sus artículos". <http://prensahistorica.mcu.es>. 


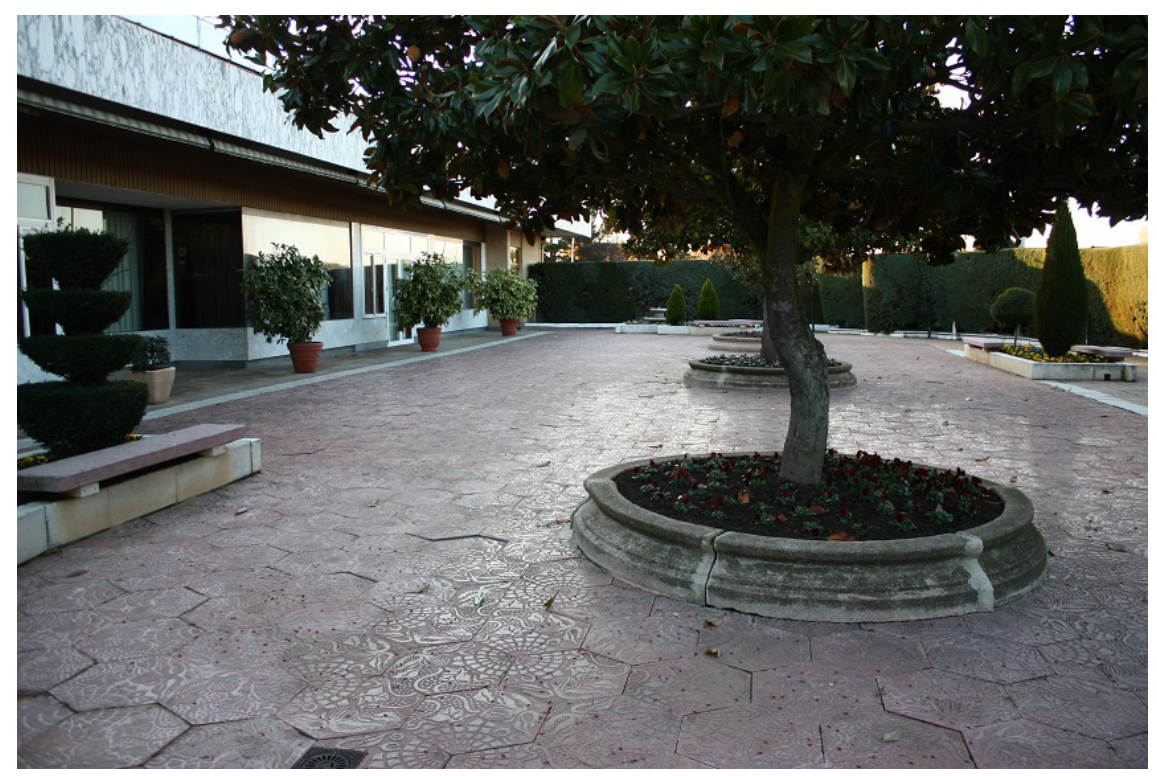

Fig.11: Vista del pavimento en la zona del jardín.

La técnica de realización de esta baldosa sigue las mismas que la realización de las piezas que hemos descrito anteriormente ${ }^{21}$, pudiendo observar en esta misma pieza un acabado rugoso -en la parte más interna de la baldosa, correspondiéndose a la parte del esgrafiado resultante- $y$ el acabado pulido de los dibujos ornamentales, ya que las diferentes capas de morteros son prensados sobre el molde de hormigón b/capa vibroprensado con relieves del cual reproduce su textura fina.

El resultado final es la baldosa que reproducimos a continuación: Una imitación al Panot hexagonal de Gaudí, es decir, una readaptación de la versión de la baldosa de Gaudí realizada por Jordi Ros a nivel industrial a mediados de los 70 y que pavimentó el paseo de Gracia en un primer momento. Según la información recopilada de los diferentes expedientes arquitectónicos consultados en el Colegio de Arquitectos de León -COAL, Delegación de Salamanca, referentes a la construcción del Hotel y a sucesivas reformas -tanto interiores como exteriores -desde el año 1955 hasta el año 1983, hemos podido observar las diferentes pautas e ideas llevadas a cabo por el arquitecto de todas estas obras, el Doctor Arquitecto D. Amando Diego Vecino.

21 Aunque en las piezas anteriormente descritas, hemos visto como el dibujo se apreciaba por el propio bajorrelieve obtenido del molde, en estas, se puede apreciar un desbaste, pulido y/o abrillantado de las mismas, obteniéndose unas superficies planas y lisas, y adquiriendo un aspecto brillante. Para ello se utilizan diferentes muelas abrasivas de grano progresivamente decreciente. Posteriormente al tratamiento superficial, las baldosas pueden ser sometidas a diversas operaciones de acabado con objeto de dotarlas de una apariencia final más atractiva y/o funcional, mediante pulidoras de cantos, perforadoras, fresadoras, fresadoras de corte tridimensional, abrasivos... 
En ninguna de las obras consultadas -alrededor de 13 expedientes- hemos encontrado documentación alguna sobre nuestro citado vestigio, excluyendo aquellos documentos legales ${ }^{22}$ en los que especifica la naturaleza y calidad de los materiales. En los documentos referidos, hemos podido observar algunas indicaciones sobre las baldosas hidráulicas, pero todas ellas de manera muy somera. Veamos más detenidamente como aparecen estos datos.

En la obra de DIEGO VECINO (1955b) ${ }^{23}$, cuando habla de las características constructivas, así habla de los diferentes pavimentos:

"Pavimentos: en general baldosa hidráulica de 40 x 40 y 30 × 30, de colores lisos a elegir. Peldaños de escalera de piedra artificial. Dos tonos en la escalera principal, un tono en la del servicio".

En el proyecto de 1964, por su parte, se observa en el epígrafe de sistema constructivo, el primer acercamiento a materiales cercanos a nuestro resto, bajo el término de baldosilla catalana:

"Pavimento de baldosa de terrazo de 40/40.- Id. De 25/25.- Pavimento de baldosín de 25/25.- Id. de baldosilla catalana en terrazas y 2 balcones.Chapado de azulejo blanco de $15 \times 15$ en sótano y oficios de piso.- Id. de color en planta baja y cuartos de baño."

Igualmente aparece de esta forma en uno de los proyectos de 1966:

\section{"Pavimento de baldosa de terrazo de 40/40 y 25/25.- baldosilla catalana en terrazas y balcones." "}

22 Hasta 1977, la construcción en España estaba regulada mediante las normas del Ministerio de la Vivienda (normas MV). En este año se aprueba el Real Decreto 1650/1977 sobre Normativa de edificación, desarrollando las Normas Básicas de la edificación (NBE), vigentes hasta 1999, momento en el cual se aprueba la Ley de Ordenación de la edificación (LOE), y se exige la redacción de un código técnico, el cual se aprobará por el Real Decreto 314/2006 entrando en vigor el Código técnico de la Edificación.

23 En esta documentación, en el anexo por titulo Pliego de condiciones facultativas y económicas que han de regir en las obras, en la capitulo II: Condiciones que deben satisfacer los materiales y su mano de obra, se lee: "e) Pavimentos: Baldosines. Articulo 17: los baldosines encarnados habrán de tener la misma confección que la teja plana anteriormente mencionada, si bien sujetos a doble compresión inicial que la que es común y corriente en aquella, presentando resistencia a la comprensión de 125 kilogramos por centímetro cuadrado. Si el baldosín fuera de cemento, después de presentar la resistencia señalada anteriormente, tendrá sus colores igualmente calibrados y entonados. Tanto unos como otros estarán perfectamente cortados, desechándose todos aquellos que presenten el mas insignificante alabeo o alguna arista desportillada. Tanto unos como otros no podrán ser empleados en obra hasta no haber trascurridos cuatro meses de su fabricación. Los dibujos y trazados del pavimento con esta clase de baldosines serán los que elija el Arquitecto Director."; y en capitulo III Ejecución de las obras Articulo 45: (...) para los pavimentos de baldosín o baldosa de arcilla, podrá aplicarse cuanto se ha dicho para los de cemento (...) 
En los últimos proyectos, de 1975 y 1983, solamente aparecerá unas pequeñas indicaciones genéricas con la frase "similar al existente", observando únicamente una pequeña referencia en el capítulo de Albañilería del pliego de condiciones:

"Baldosín catalán.- Se fabricaran con tierras arcillosas, cocidas a temperaturas inferior a la vitrificación.- Serán fabricados a maquina por doble laminación.- Serán impermeables, no presentarán alabeos, ni fisuras."

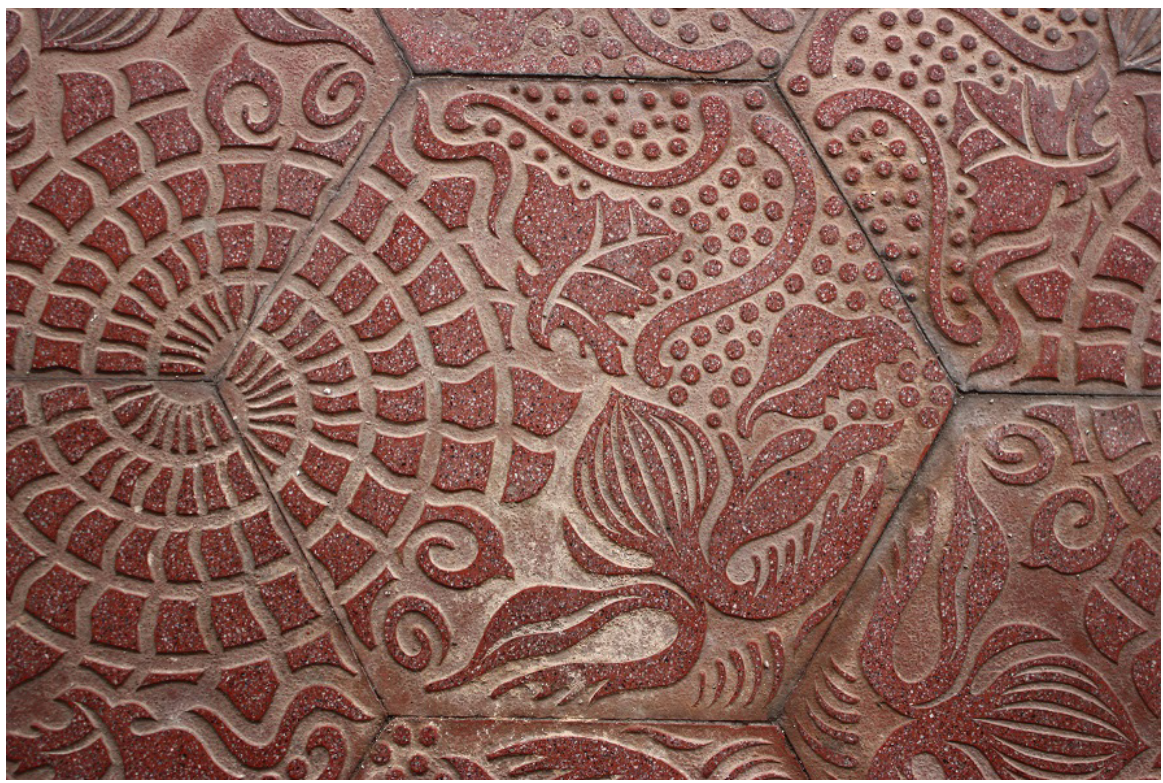

Fig.12: Vista detalle del pavimento.

Creemos por tanto, que a raíz de la obra de Barcelona, multitud de arquitectos se interesaron en esta baldosa para sus diferentes obras, y a tenor de lo encontrado, uno de los interesados fue D. Amando Diego Vecino.

\section{Conclusiones}

Antoni Gaudí y su obra se han convertido en icono. Sus palabras, sus geniales proyectos arquitectónicos y su talante se celebraron con gran éxito en el año 2002, dedicado a Gaudí en el 150 aniversario de su nacimiento. Arquitectura, ingeniería y diseño industria ${ }^{24}$ bajo el sello de este visionario fueron homenajeados y dados

24 Según Oriol Pibernat "Es muy probable que el Año Gaudí nos recuerde o descubra, según los casos, a un Gaudí diseñador. En primer lugar porque su obra, al igual que el movimiento modernista en el que se inscribe de forma original, se sitúa en el centro de las relaciones entre arte y técnica, artesanado e industria, ornamento y estructura, creación individualizada y serie, racionalidad constructiva y voluntad expresiva, estilos y nuevos lenguajes, urbanismo y domesticidad, etc., 
a conocer tanto al mundo como a los habitantes de su propio país de origen con el detalle y la admiración que merece.

Sin embargo, muchas veces tal admiración desemboca en, por ejemplo, una desmesurada utilización del trencadís en monumentos de posterior factura o, en el peor delos casos, en las Ilamadas mercaderías culturales ${ }^{25}$, souvenirs, objetos decorativos, cerámicas, gráficas aplicadas,etc. algunas de ellas con dudoso gusto o de existencia inapropiada.

En el caso que nos ocupa -las baldosas dispuestas en el Hotel Regiocontemplamos su presencia como efectivamente inapropiada dentro del conjunto urbanístico, sin el más mínimo testimonio a su concepción, forma autor o cualquier otra referencia a su diseño y sentido original. Se convierten en una imitación, un falso testimonio, Gaudí no estuvo aquí.

Conocer, entender y respetar nuestro Patrimonio implica investigar, proteger y divulgar por parte de los gestores de Patrimonio que somos.

La intención de este artículo es dar a conocer un resto paradigmático dentro del mundo de la decoración urbana. La perspectiva que se nos abre es bastante grande, no solo por su valor estético sino por la información cultural que ofrece. La viabilidad de realizar catálogos, muestras o cualquier otro tipo de acción, es una propuesta novedosa dentro del patrimonio de una ciudad viva que debe actuar por/para/con el ciudadano, y aquellos objetos mas próximos en el tiempo, no deja de ser parte de nuestro patrimonio. Objetos que se escapan de los discursos preestablecidos: baldosas hidráulicas, placas de seguro, de aseguradoras de incendios, del Ministerio de la Vivienda, mobiliario urbano de carácter industrial que pasa desapercibido. Etnografía urbana, arqueología industrial.

elementos que, en su conjunto, configuran las bases del diseño moderno. Pero, además, porque Daniel Giralt-Miracle, comisario del Año Gaudí, ha sido un propagador entusiasta de una visión de Gaudí que ha hecho hincapié tanto en los aspectos arquitectónicos como en los de diseño, y que descubre en el maestro de Reus a un diseñador más moderno que el más moderno de los diseñadores." <http://www.bcn.es/publicacions/b_mm/ebmm58/bmm58_qc65.htm>

25 Entre estas mercancías culturales tenemos que romper una lanza en una serie de productos, que podríamos calificar como objetos de reproducción, cita u homenaje, que lanzan un destello de luz dentro del oscuro mundo de la tienda de regalos, a sabiendas de que existen todos y cada uno de los productos que se imaginen con la iconografía propia del autor y de sus obras. En este caso concreto, nos limitaremos a comentar dos de ellos que nos parecen fuera de lo común, por una parte la reproducción propia de la baldosa hexagonal de Gaudí < http://www.gaudibarcelo nashop. com/6-baldosas > realizadas en diversos colores y con una serie de diseños diferentes, que pueden ser utilizadas tanto para uso tradicional como decorativo; y el juego de mesa de las baldosas de Gaudí, <www.gaudipanot.com> creado por Oriol Comas junto a Jep Ferret, donde cada pieza del juego es una baldosa en miniatura, con diferentes combinaciones de colores en cada una de sus tres partes. Para el II Encuentro Nacional de Juegos de Mesa realizado en Córdoba en $2006<$ http:// jugamostodos.awardspace.com/index.php?option=com_content\&task=view\&id=3\&ltem id=5> se observó la versión gigante -a escala 1:1, del tamaño original de la baldosa- para jugar en la calle que se había realizado años antes. 


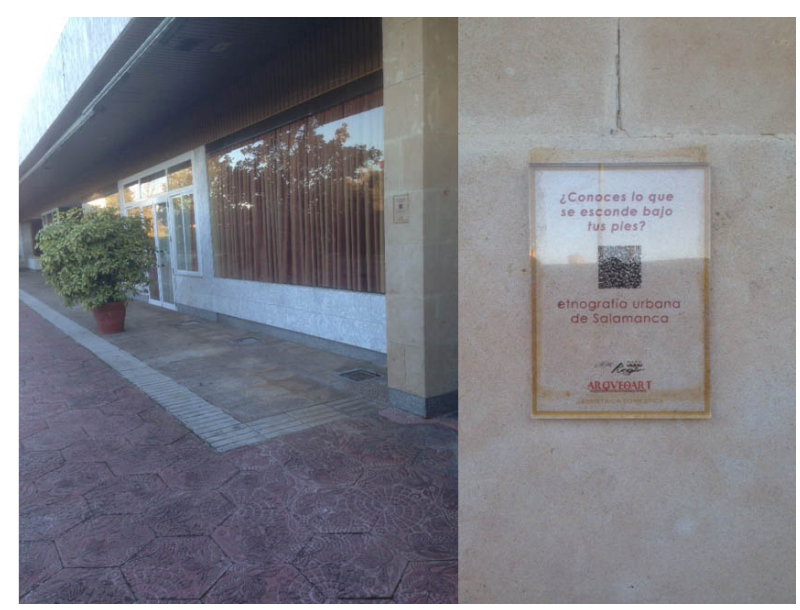

Fig. 13. Placa informativa ${ }^{26}$.

La Revolución Industrial nos queda ya lejos, es conveniente que comencemos a recabar la información que demandarán las generaciones futuras sobre las fábricas, sus mecanismos, sus condiciones, sus diseñadores, sus productos. El uso de los mismos en la urbe, en la ciudad donde habitamos, nos hará comprender aspectos humanos que pasan desapercibidos por cotidianos pero que forman parte de nuestras costumbres y hábitos. La posibilidad de visionar esta singular pieza de diseño industrial, en una provincia donde el modernismo dejó pocas referencias de su paso por la capital, nos parece destacable y digna de realizar este estudio que, aunque la haya determinado como una imitación o falsa baldosa, nos conduce hacia un mejor conocimiento de nuestro entorno inmediato.

\section{Bibliografía}

ALSINA i CATALÁ, Claudi; (2004): Manual de recursos didácticos de Geometría para colaboradores del Gabinet Gaudí. Departamento de Estructuras en arquitectura. E.T.S. de Arquitectura en Barcelona.

BALLART, J.; (1997): El patrimonio Histórico y Arqueológico. Valor y uso. Ed. Ariel. Barcelona.

26 A raíz de la presentación del proyecto en diferentes congresos y reuniones, y su posicionamiento en ambientes virtuales con una aceptación muy recomendable, conseguimos permiso por parte de la dirección del hotel de la colocación de una placa informativa sobre el pavimento en una de las dependencias del propio hotel, donde mediante un código QR, el visitante, turista, comensal o usuario, puede consultar libremente la documentación sobre el citado resto, es decir, puede leer -del mismo modo que lo estás haciendo tú, lector- este pequeño trabajo divulgativo y de investigación. 
BALLART, J.; JUAN i TRESSERAS, J.; (2001): Gestión del Patrimonio Cultural. Ed. Ariel. Barcelona.

BONET CORREA, Antonio; (Coord.) (1972): Historia de la Artes aplicadas e Industriales en España. Cátedra. Madrid.

DIEGO VECINO, Amando; (1955a): Anteproyecto de Hotel turismo en Santa Marta de Tormes (Salamanca). Informe inédito depositado en el Colegio de Arquitectos de León. Delegación de Salamanca.

DIEGO VECINO, Amando; (1955b): Proyecto de ampliación y reforma del restaurante Jardín-Regio para su trasformación en hotel de turismo "JardínRegio" en Santa Marta de Tormes (Salamanca). Informe inédito depositado en el Colegio de Arquitectos de León Delegación de Salamanca.

DIEGO VECINO, Amando; (1964): Anteproyecto de Hotel de turismo en el km. 204,640 de la carretera de Villacastín a Vigo -Santa Marta de Tormes (Salamanca). Informe inédito depositado en el Colegio de Arquitectos de León. Delegación de Salamanca.

DIEGO VECINO, Amando; (1966): Anteproyecto de ampliación de un piso en hotel de turismo "1a A" en el km. 204,640 de la carretera de Villacastín a Vigo en Santa Marta de Tormes (Salamanca). Informe inédito depositado en el Colegio de Arquitectos de León Delegación de Salamanca.

DIEGO VECINO, Amando; (1975): Proyecto de ampliación de semisótano y planta baja, en el Hotel "Regio" de Santa Marta de Tormes (Salamanca). Informe inédito depositado en el Colegio de Arquitectos de León Delegación de Salamanca.

DIEGOVECINO, Amando; (1983): Proyecto de ampliación de comedor "Lazarillo" dos tenedores en Hotel Regio, sito en la carretera de Villacastín a Vigo km. 204,640 en Santa Marta de Tormes (Salamanca). Informe inédito depositado en el Colegio de Arquitectos de León Delegación de Salamanca.

ESPAÑA. Ley 38/1999, de 5 de noviembre, de Ordenación de la Edificación. Boletín oficial del estado: 6 de noviembre de 1999. no. 266. Pp. 38925-38934. ESPAÑA. Real Decreto 1650/1977, de 10 de junio, sobre Normativa de la edificación. Boletín oficial del Estado: 9 de julio de 1977. no 163. Pp. 15443- 15444.

ESPAÑA. Real Decreto 314/2006, de 17 de marzo, por el que se aprueba el código Técnico de la Edificación. Boletín oficial del Estado: 28 de marzo de 2006. no 74. Pp. 11816-11831.

FREIXA, Mireia; (1982): El modernismo en España. Cátedra. Madrid.

GIRALT-MIRACLE, Daniel; (2002): Gaudí. La búsqueda de la forma. Espacio, geometría, estructura y construcción. Ayuntamiento de Barcelona Institut de Cultura Sociedad Estatal para la Acción Cultural Exterior. Ayuntamiento de Barcelona. Institut de Cultura. Lunwerg Editores SA. 
HERNANDEZ NAVARRO, Mario Arturo; (2006) Barcelona Tile designs. Amsterdam Ed. The Pepin Press. Agile Rabbit Editions.

LINAREJOS CRUZ, María; FERNÁNDEZ-POSSE, Dolores; HUMANES, Alberto; DE LA MATA, Ramón; (2002); "El Plan Nacional de Patrimonio Industrial" Actas de las III Jornadas Internacionales de Patrimonio Industrial, junio 2001. INCUNA. Asociación de Arqueología Industrial, Colección. "Los ojos de la memoria" volumen no 2. Patrimonio Industrial. Lugares de la memoria: Proyectos de reutilización en industrias culturales, turismo y museos. Gijón.

MORALES MIRANDA, J.; (1998): Guía practica para la interpretación del patrimonio. El arte de acercar el legado natural y cultural al público visitante. Junta de Andalucía. Conserjería de Cultura. Sevilla.

PERLA, Antonio; (1988): Cerámica aplicada en la Arquitectura Madrileña. Comunidad de Madrid. Conserjería de Política Territorial. Dirección general de Arquitectura. Madrid.

PUIG i BOADA, Isidre; (2004): El pensament de Gaudí. Ed. Dux, Barcelona.

QUEROL, M․ Ángeles; MARTINEZ DIAZ, B.; (1996): La gestión del Patrimonio Arqueológico en España. AUT 161. Madrid.

RASCON MARQUÉS, Sebastián; (2002): "El mundo en sus manos. O cómo utilizar las nuevas tecnologías en la difusión del patrimonio arqueológico". Actas del $20^{\circ}$ Congreso Internacional de Musealización de Yacimientos Arqueológicos. Museo d'Historia de la Ciutat. Barcelona.

RENFREW, C.; BAHN, P.; (1998): Arqueología. Teorías, métodos y practica. Ed. Akal. Madrid.

REYES TELLEZ, Francisco; (2004): "El patrimonio arqueológico Industrial en la ciudad Histórica." Anales de arqueología Cordobesa no 15. Pp. 83-99. ed. Universidad de Córdoba. Facultad de Filosofía y letras. Córdoba.

SANTACANA, Joan; HERNANDEZ, Xavier; (1999): Enseñanza de la Arqueología y la Prehistoria. Colección Educación serie materiales 1. Ed. Mileno. Lleida.

TARRAGONA, Josep Maria; (1999): Gaudí, biografia de I'artista. Ed. Proa, Barcelona.

TILDEN, Freeman; (1957). Interpreting Our Heritage. The University of North Carolina Press, Chapel Hill. AIP; (2007): La interpretación de nuestro Patrimonio. Trad. de la Asociación para la Interpretación del Patrimonio.

VV.AA.; (2000): Turismo Cultural. El patrimonio Histórico como fuente de riqueza. Ed.Fundación del Patrimonio Histórico de Castilla y León. Valladolid.

VV.AA.; (2002): Gaudí 2002. Miscel·lània. Ed. Planeta, Barcelona.

VV.AA.; (2005): Plan PAHIS 2004-2012 del Patrimonio de Castilla y León. Ed. Junta de Castilla y León. Conserjería de Cultura y Turismo. Valladolid. 
VV.AA.; (2007): ACTAS Accesibilidad y Patrimonio. Yacimientos arqueológicos, cascos históricos, jardines y monumentos. Ed. Junta de Castilla y León. Conserjería de Cultura y Turismo. Valladolid.

ZERBST, Rainer; (1985): Antoni Gaudí. Ed. Taschen, Köln. 